\title{
THE PRINTED VERSION OF THE HIPPARCOS INPUT CATALOGUE
}

\author{
C.TURON, D.MORIN, A.GOMEZ, F.CRIFO, F.ARENOU, A.SELLIER, \\ M.MAROUARD \\ DASGAL and URA 335 du CNRS - Observatoire de Paris. F-92195 MEUDON
}

\begin{abstract}
The data selected for publication and the stellar content of the 118,000 stars constituting the observing programme of the Hipparcos satellite are briefly described.
\end{abstract}

The printed version of the Hipparcos Input Catalogue does NOT give results obtained by the satellite itself. It contains, for the 118,000 programme stars included:

- The basic data delivered to European Space Agency to operate the satellite (J2000 positions, proper motions, Hipparcos magnitude, colour, indications on multiplicity and variability). These data were selected among existing ones, or newly observed by the INCA Consortium when existing data were of poor quality.

- Additional data, gathered or newly established by the Consortium, of great interest to the astronomers, such as numerous cross-identifications, B1950 positions, spectral type, $V$ magnitude and $B-V$ colour, parallax and radial velocity when available, more data on variability and multiplicity, sources of data, notes.

- Five Annexes with more details: multiplicity (data on each component); finding charts for the faintest stars (10,000 objects), and for stars in the galactic clusters (1600 objects) and in the Magellanic Clouds; list of all Proposals made to ESA.

The 118,000 programme stars, all brighter than $\mathrm{V}=12.5$, are rather evenly distributed over the sky, with a slight enhancement near the galactic plane; $85 \%$ are closer than 500 parsecs; the peak of magnitude distribution is around 9; all stars brighter than 7.3 are included. Accuracies: positions, all better than $1.5 \mathrm{arcsec}$, with a rms value of 0.3 arcsec; magnitudes, all better than 0.5 mag.; half of them better than 0.05 mag.

Detailed descriptions of the Input Catalogue construction may be found in the references below. The publication is expected for fall 1991 and will have the reference ESA SP-1136. A tape version will be available at CDS.

\section{References}

Proceedings of Aussois Colloquium, 1985, ESA SP-234.

Proceedings of Sitges Colloquium, 1988, Torra \& Turon (eds).

The Hipparcos mission, pre-launch status, 1989, ESA SP-1111 vol.II.

Special issue of 'Astron.\& Astroph. Suppl.' on first Hipparcos results, in press. 